\title{
Adaptive fuzzy synchronization of uncertain fractional-order chaotic systems with different structures and time-delays
}

\author{
Xiaoli Qin ${ }^{1,2}$, Shenggang Lij ${ }^{*}$ and Heng Liü
}

"Correspondence:

shengganglinew@126.com

${ }^{1}$ College of Mathematics and

Information Science, Shaanxi

Normal Universtiy, Xi'an, China

Full list of author information is

available at the end of the article

\section{Springer}

\begin{abstract}
To achieve synchronization of uncertain fractional-order chaotic systems with time-delays, an adaptive fuzzy controller with integer-order parameter adaptive laws, is established. Unknown nonlinear functions and uncertain external disturbances are approximated by fuzzy logic systems. Adaptive laws are designed to adjust corresponding parameters in the controller. The proposed controller guarantees that the synchronization error of the system converges to a small enough region of the origin by making use of quadratic Lyapunov functions in the stability analysis, and the boundedness of all signals in the closed-loop. Finally, simulation studies have been provided to verify the effectiveness of the proposed methods.
\end{abstract}

Keywords: Adaptive fuzzy control; Fractional-order chaotic system; Systems with time-delay

\section{Introduction}

Although fractional calculus has a history as long as the integer-order calculus, it has received much attention only in recent several decades because it has been shown that the fractional calculus can provide real-world systems some useful properties, such as hereditary and memory [1-12]. With the supplement and perfection of fractional theory, fractional calculus shows better application value and development prospects than the integer-order one. Fractional-order calculus provides not only new methods of mathematics for actual systems, but also gives a more comprehensive mathematical model. Fractional-order systems have shown an attractive property of obvious performance improvement in the area of image encryption, security communications [13] and biological medicine [14]. Therefore, researching the synchronization problem of fractional-order chaotic systems is very necessary. By using a lot of control approaches, such as active control, sliding mode control, adaptive fuzzy control, neural network control, $H_{\infty}$ control, a large quantity of works as regards the synchronization issue of fractional-order chaotic systems, have been reported in Refs. [15-21]. It should be mentioned that in the above literature, the time-delays were not considered.

On the other hand, due to the mechanical, physical and economic impact, the delay phenomenon often appears in actual systems [22-28]. The dynamic performance of systems at the presence of time-delay characteristics, such as motion orbit of systems and fluctua-

(c) The Author(s) 2019. This article is distributed under the terms of the Creative Commons Attribution 4.0 International License (http://creativecommons.org/licenses/by/4.0/), which permits unrestricted use, distribution, and reproduction in any medium, provided you give appropriate credit to the original author(s) and the source, provide a link to the Creative Commons license, and indicate if changes were made. 
tion of stability, will generate noticeable changes. Consequently, it is very hard to synchronize uncertain fractional-order chaotic systems with time-delays. Up to now, there exist only a few approaches that consider the synchronization of fractional-order chaotic systems with time-delays. In Ref. [25], the synchronization of fractional-order systems with time-delays was solved, but it cannot deal with the synchronization in fractional-order systems with uncertain parameters. In Ref. [26], a feedback control method was used for switched time-delay systems with nonlinear disturbances. However, the major disadvantage of the feedback control is that the synchronization of systems with unknown parameters is not considered. In Ref. [29], the synchronization of fractional-order chaotic systems with time-delays was achieved by designing a sliding mode controller, where the external disturbances were not considered and one needed to known the exact upper bounds of unknown terms. Besides, their methods cannot be used to control fractional-order chaotic systems with both different structures and time-delays. In Ref. [30], the pulse synchronization of fractional-order chaotic systems with time-delays was finished. Nonetheless, the designed controller is not able to achieve the synchronization of systems with uncertain terms or systems with external disturbances. Thereby, the synchronization problem for fractional-order chaotic systems with time-delays, uncertain terms and external disturbances still needs to be further investigated.

Nevertheless, real-world systems usually suffer from system uncertainties, such as, sensor errors, unknown external disturbances, system modeling errors, which will decrease the control performance if they are not well handled [22,31-36]. As is well known, fuzzy logic systems can be utilized to control nonlinear systems with unknown structure due to the fact that it does not need an accurate system model and it can take advantage of human expert knowledge. The effectiveness of this control method has been indicated in the integrator-order system control. To handle the fuzzy approximation error, some other control methods, for example, sliding mode control, $H_{\infty}$ control should be used together with adaptive fuzzy control. Recently, the adaptive fuzzy control has been extended to control fractional-order nonlinear systems, for example, in [2, 20, 21, 37-40]. However, to the best of our knowledge, the adaptive fuzzy control for fractional-order nonlinear systems with time-delays has rarely been investigated up to now.

Motivated by above discussion, to achieve the synchronization problem of unknown fractional-order chaotic systems with time-delays, this paper designs a controller that can be used to eliminate time-delay characteristics, nonlinear terms and external disturbances in nonlinear systems. Considering systems with unknown parameters and external disturbances, this paper studies the synchronization of unknown fractional-order chaotic systems with different structures and time-delays based on adaptive fuzzy control [37, 41-43]. Our main contributions are given as follows: (1) a fuzzy system is applied to approximate plant uncertainty, which contains time-delay state variables, unknown nonlinear terms and uncertain external disturbances. It should be mentioned that proposed controller works well even the system models are fully unknown; (2) the paper designs integer-order parameter adaptive laws that achieve adaptive adjustment of the controller based on Lyapunov stability theorem; (3) this paper strictly justifies the stability of the system by constructing and making use of quadratic Lyapunov function. Synchronization between fractional-order Liu system with time-delay and fractional-order Chen system with time-delay is achieved in a numerical simulation. 
The framework of this paper is as follows. Section 2 introduces some basic knowledge and many basic conclusions about fractional calculus and problem description. Controller design methods and unique result in the paper are in Sect. 3 and Sect. 4, separately. Section 5 is numerical simulation. Finally, summing up the work and making some predictions are given in Sect. 6.

\section{Preliminaries and problem description}

\subsection{Fractional calculus}

Noting that the initial value of the Caputo fractional-order derivatives has the same form as that of the integer-order system which has better engineering applications, this paper will use this definition. The $\mu$ th fractional-order integral can be defined as:

$$
{ }_{0}^{C} D_{t}^{-\mu} f(t)=\frac{1}{\Gamma(\mu)} \int_{0}^{t}(t-\tau)^{\mu-1} f(\tau) d \tau
$$

where the $\Gamma(\cdot)$ function is

$$
\Gamma(z)=\int_{0}^{\infty} t^{z-1} e^{-t} d t
$$

The $\mu$ th Caputo derivatives is defined as

$$
{ }_{0}^{C} D_{t}^{\mu} f(t)=\frac{1}{\Gamma(n-\mu)} \int_{0}^{t}(t-\tau)^{n-\mu-1} f^{(n)}(\tau) d \tau,
$$

where $n$ is an integer satisfying $n-1 \leq \mu<n$. For the sake of brevity, we will use signals ${ }_{0} D_{t}^{\mu}$ and ${ }_{0} D_{t}^{-\mu}$ to, respectively, represent ${ }_{0}^{C} D_{t}^{\mu}$ and ${ }_{0}^{C} D_{t}^{-\mu}$.

The Laplace transform of Caputo fractional derivatives (3) is expressed by [1]

$$
\mathcal{L}\left({ }_{0} D_{t}^{\mu} f(t)\right)=\int_{0}^{\infty} e^{-s t} D_{t}^{\mu} f(t) d t=s^{\mu} F(s)-\sum_{k=0}^{n-1} s^{\mu-k-1} f^{(k)}(0) .
$$

Obviously, when $0<\mu<1, \mathscr{L}\left({ }_{0} D_{t}^{\mu} f(t)\right)=s^{\mu} F(s)-s^{\mu-1} f(0)$.

For conveniently discussing, we assume $\mu \in(0,1)$ in the rest of this paper. The following conclusions will be used.

Definition 1 ([44]) The Mittag-Leffler function with two parameters can be written as

$$
E_{\mu, \xi}(z)=\sum_{t=0}^{\infty} \frac{z^{t}}{\Gamma(\mu t+\xi)},
$$

where $\mu, \xi>0$, and $z \in C$. Obviously, $E_{1,1}(z)=e^{z}$.

The Laplace transform of the Mittag-Leffler function is [44]

$$
\mathcal{L}\left\{t^{\xi-1} E_{\mu, \xi}\left(-b t^{\mu}\right)\right\}=\frac{s^{\mu-\xi}}{s^{\mu}+b} .
$$


Definition 2 ([45]) The convolution of functions $f$ and $g$ is defined as

$$
f * g=\int_{0}^{t} f(\tau) g(t-\tau) d \tau
$$

where $t \in[0,+\infty)$.

Lemma 1 ([46]) If $v(t) \in C^{1}[0, h](h>0)$, then the following equality holds:

$$
{ }_{0} D_{t 0}^{\mu} D_{t}^{-\mu} v(t)=v(t) \quad(t \in[0, h]) .
$$

Lemma 2 ([46]) If $v(t) \in C^{1}[0, h](h>0)$, then we have

$$
{ }_{0} D_{t 0}^{\mu} D_{t}^{\beta} v(t)={ }_{0} D_{t 0}^{\beta} D_{t}^{\mu} v(t)={ }_{0} D_{t}^{\mu+\beta} v(t)=\dot{v}(t) \quad(t \in[0, h]),
$$

where $\mu, \beta \in \mathbb{R}^{+}$and $\mu+\beta=1$.

\subsection{Description of fuzzy systems}

A fuzzy logic system includes four parts, i.e., the knowledge base, the fuzzifier, the fuzzy inference engine basing on the fuzzy rules, and the defuzzifier. The $j$ th fuzzy rule is expressed by $\mathcal{R}^{(j)}$ : if $x_{1}$ is $E_{1}^{j}, x_{2}$ is $E_{2}^{j}, \ldots, x_{n}$ is $E_{n}^{j}$, then $\hat{f}(\boldsymbol{x}(t))$ is $C^{j}$ where $(j=1,2, \ldots, N)$, $\boldsymbol{x}(t)=\left[x_{1}(t), x_{2}(t), \ldots, x_{n}(t)\right]^{T} \in \mathbb{R}^{n}$ and $\hat{f}(\boldsymbol{x}(t)) \in \mathbb{R}$ are, respectively, the input and the output of fuzzy logic systems. $E_{i}^{j}$ and $C^{j}(i=1,2, \ldots, n)$ are fuzzy sets belonging to $\mathbb{R}$. The output of fuzzy logic systems can be expressed by

$$
\hat{f}(\boldsymbol{x}(t))=\frac{\sum_{j=1}^{N} \theta_{j}(t)\left[\prod_{i=1}^{n} \mu_{E_{i}^{j}}\left(x_{i}(t)\right)\right]}{\sum_{j=1}^{N}\left[\prod_{i=1}^{n} \mu_{E_{i}^{j}}\left(x_{i}(t)\right)\right]},
$$

where $\theta_{j}(t)$ is a value where fuzzy membership function $\mu_{C^{j}}$ is maximum. Generally, we can consider that $\mu_{C^{j}}\left(\theta_{j}(t)\right)=1$, and the fuzzy basic function is $\varphi_{j}(x(t))=\frac{\prod_{i=1}^{n} \mu_{i_{i}^{j}}\left(x_{i}(t)\right)}{\sum_{j=1}^{N}\left[\prod_{i=1}^{n} \mu_{E_{i}^{j}}\left(x_{i}(t)\right)\right]}$. Let $\boldsymbol{\varphi}(\boldsymbol{x}(t))=\left[\varphi_{1}(\boldsymbol{x}(t)), \varphi_{2}(\boldsymbol{x}(t)), \ldots, \varphi_{N}(\boldsymbol{x}(t))\right]^{T}, \boldsymbol{\theta}(t)=\left[\theta_{1}(t), \theta_{2}(t), \ldots, \theta_{N}(t)\right]^{T}$, thus, the output of fuzzy logic systems can be written as

$$
\hat{f}(\boldsymbol{x}(t))=\boldsymbol{\theta}^{T}(t) \varphi(\boldsymbol{x}(t)) .
$$

Theorem 1 Suppose that $h(\boldsymbol{x})$ is a continuous function defined on a compact set $\Omega$, then, for any constants $\varepsilon>0$, there exists a fuzzy logic system approximating function $\hat{f}(\boldsymbol{x})$ with the form (11) as

$$
\sup _{\Omega}\left|h(\boldsymbol{x})-\hat{\boldsymbol{\theta}}^{T} \boldsymbol{\varphi}(\boldsymbol{x})\right| \leq \varepsilon
$$

where $\hat{\boldsymbol{\theta}}$ is the estimator of optimal vector $\boldsymbol{\theta}^{*}$. 


\subsection{Problem description}

Consider the fractional-order drive and response chaotic systems with time-delays defined by

$$
\begin{aligned}
& { }_{0} D_{t}^{\mu} \boldsymbol{x}(t)=\boldsymbol{f}\left(\boldsymbol{x}(t), \boldsymbol{x}\left(t-\tau_{1}\right)\right)+\triangle \boldsymbol{h}_{1}(\boldsymbol{x}(t))+\boldsymbol{D}_{1}(t), \\
& { }_{0} D_{t}^{\mu} \boldsymbol{y}(t)=\boldsymbol{g}\left(\boldsymbol{y}(t), \boldsymbol{y}\left(t-\tau_{2}\right)\right)+\Delta \boldsymbol{h}_{2}(\boldsymbol{y}(t))+\boldsymbol{D}_{2}(t)+\boldsymbol{U}(t),
\end{aligned}
$$

where $\boldsymbol{x}(t)=\left[x_{1}(t), x_{2}(t), \ldots, x_{n}(t)\right]^{T} \in R^{n}$ and $\boldsymbol{y}(t)=\left[y_{1}(t), y_{2}(t), \ldots, y_{n}(t)\right]^{T} \in R^{n}$ are, respectively, the state variables of the drive system and response system, $x\left(t-\tau_{1}\right)=\left[x_{1}(t-\right.$ $\left.\left.\tau_{1}\right), x_{2}\left(t-\tau_{1}\right), \ldots, x_{n}\left(t-\tau_{1}\right)\right]^{T}$ and $\boldsymbol{y}\left(t-\tau_{2}\right)=\left[y_{1}\left(t-\tau_{2}\right), y_{2}\left(t-\tau_{2}\right), \ldots, y_{n}\left(t-\tau_{2}\right)\right]^{T} \in R^{n}$ are the state variables with time-delays, $f, g: R^{n} \rightarrow R^{n}$ are uncertain nonlinear continuous functions, $\Delta \boldsymbol{h}_{1}(\boldsymbol{x}(t))=\left[\Delta h_{11}, \Delta h_{12}, \ldots, \Delta h_{1 n}\right]^{T}$ and $\Delta \boldsymbol{h}_{2}(\boldsymbol{y}(t))=\left[\Delta h_{21}, \Delta h_{22}, \ldots, \Delta h_{2 n}\right]^{T} \in R^{n}$ are unknown nonlinear terms, $D_{1}(t)=\left[d_{11}(t), d_{12}(t), \ldots, d_{1 n}(t)\right]^{T}$ and $\boldsymbol{D}_{2}(t)=\left[d_{21}(t), d_{22}(t)\right.$, $\left.\ldots, d_{2 n}(t)\right]^{T} \in R^{n}$ are unknown external disturbances, and $\boldsymbol{U}(t)=\left[u_{1}(t), u_{2}(t), \ldots, u_{n}(t)\right]^{T} \in$ $R^{n}$ is the control input.

\section{Controller design methods}

The synchronization error is defined as $\boldsymbol{e}(t)=\boldsymbol{y}(t)-\boldsymbol{x}(t)$. In this paper, the control objective is to design an adaptive controller such that the synchronization error will be arbitrarily small eventually.

The dynamic equation of synchronization error can be expressed by

$$
\begin{aligned}
{ }_{0} D_{t}^{\mu} \boldsymbol{e}(t)= & { }_{0} D_{t}^{\mu}(\boldsymbol{y}(t)-\boldsymbol{x}(t)) \\
= & { }_{0} D_{t}^{\mu} \boldsymbol{y}(t)-{ }_{0} D_{t}^{\mu} \boldsymbol{x}(t) \\
= & \boldsymbol{g}\left(\boldsymbol{y}(t), \boldsymbol{y}\left(t-\tau_{2}\right)\right)-\boldsymbol{f}\left(\boldsymbol{x}(t), \boldsymbol{x}\left(t-\tau_{1}\right)\right)+\triangle \boldsymbol{h}_{2}(\boldsymbol{y}(t))-\triangle \boldsymbol{h}_{1}(\boldsymbol{x}(t)) \\
& +\boldsymbol{D}_{2}(t)-\boldsymbol{D}_{1}(t)+\boldsymbol{U}(t) .
\end{aligned}
$$

Denote $\boldsymbol{F}(\boldsymbol{z}(t))=\boldsymbol{g}\left(\boldsymbol{y}(t), \boldsymbol{y}\left(t-\tau_{2}\right)\right)-\boldsymbol{f}\left(\boldsymbol{x}(t), \boldsymbol{x}\left(t-\tau_{1}\right)\right)+\Delta \boldsymbol{h}_{2}(\boldsymbol{y}(t))-\Delta \boldsymbol{h}_{1}(\boldsymbol{x}(t))+\boldsymbol{D}_{2}(t)-\boldsymbol{D}_{1}(t)=$ $\left[F_{1}, F_{2}, \ldots, F_{n}\right]^{T}$, where $\boldsymbol{z}(t)$ is a function about $\boldsymbol{x}(t)$ and $\boldsymbol{y}(t)$, then Eq. (15) can be written as

$$
{ }_{0} D_{t}^{\mu} \boldsymbol{e}(t)=\boldsymbol{F}(\boldsymbol{z}(t))+\boldsymbol{U}(t)
$$

The unknown function $\boldsymbol{F}(\boldsymbol{z}(t))$ can be approximated by the fuzzy logic system as

$$
\hat{F}_{i}\left(\theta_{i}(t), \boldsymbol{z}(t)\right)=\theta_{i}(t)^{T} \varphi_{i}(\boldsymbol{z}(t)), \quad i=1,2, \ldots, n,
$$

where $\varphi_{i}(\boldsymbol{z}(t))$ is a fuzzy basic function, and $\theta_{i}(t)$ is an adjustable parameter of the fuzzy logic system. Let an optimal estimated parameter of the fuzzy logic system be $\theta_{i}^{*}$ (where $\theta_{i}^{*}=\arg \sup _{t}\left|F_{i}(\boldsymbol{z}(t))-\hat{F}_{i}\left(\theta_{i}(t), \boldsymbol{z}(t)\right)\right|$, and $\theta_{i}^{*}$ is generally a constant vector). Suppose that the errors of optimal parameter and the optimal estimated errors are separately

$$
\begin{aligned}
& \tilde{\theta}_{i}(t)=\theta_{i}(t)-\theta_{i}^{*}, \\
& \varepsilon_{i}(\boldsymbol{z}(t))=F_{i}(\boldsymbol{z}(t))-\hat{F}_{i}\left(\theta_{i}^{*}, \boldsymbol{z}(t)\right) .
\end{aligned}
$$


From Refs. $[47,48]$ and Theorem 1 , the estimated error of the fuzzy logic system is assumed to be bounded, i.e. $\left|\varepsilon_{i}(\boldsymbol{z}(t))\right| \leq \varepsilon_{i}^{*}\left(\varepsilon_{i}^{*}>0\right.$ is an uncertain constant). Let $\boldsymbol{\varepsilon}(\boldsymbol{z}(t))=\left[\varepsilon_{1}(\boldsymbol{z}(t)), \varepsilon_{2}(\boldsymbol{z}(t)), \ldots, \varepsilon_{n}(\boldsymbol{z}(t))\right]^{T}, \boldsymbol{\varepsilon}^{*}=\left[\varepsilon_{1}^{*}, \varepsilon_{2}^{*}, \ldots, \varepsilon_{n}^{*}\right]^{T}, \boldsymbol{\theta}^{*}=\left[\theta_{1}^{*}, \theta_{2}^{*}, \ldots, \theta_{n}^{*}\right]^{T}$ and $\boldsymbol{\theta}(t)=\left[\theta_{1}(t), \theta_{2}(t), \ldots, \theta_{n}(t)\right]^{T}$, the estimated error of the unknown nonlinear function can be expressed as

$$
\begin{aligned}
\boldsymbol{F}(\boldsymbol{z}(t))-\hat{\boldsymbol{F}}(\boldsymbol{\theta}(t), \boldsymbol{z}(t)) & =\boldsymbol{F}(\boldsymbol{z}(t))-\hat{\boldsymbol{F}}\left(\boldsymbol{\theta}^{*}, \boldsymbol{z}(t)\right)+\hat{\boldsymbol{F}}\left(\boldsymbol{\theta}^{*}, \boldsymbol{z}(t)\right)-\hat{\boldsymbol{F}}(\boldsymbol{\theta}(t), \boldsymbol{z}(t)) \\
& =\boldsymbol{\varepsilon}(\boldsymbol{z}(t))+\hat{\boldsymbol{F}}\left(\boldsymbol{\theta}^{*}, \boldsymbol{z}(t)\right)-\hat{\boldsymbol{F}}(\boldsymbol{\theta}(t), \boldsymbol{z}(t)) \\
& =\boldsymbol{\varepsilon}(\boldsymbol{z}(t))-\left(\boldsymbol{\theta}(t)-\boldsymbol{\theta}^{*}\right)^{T} \boldsymbol{\varphi}(\boldsymbol{z}(t)) \\
& =\boldsymbol{\varepsilon}(\boldsymbol{z}(t))-\tilde{\boldsymbol{\theta}}(t)^{T} \boldsymbol{\varphi}(\boldsymbol{z}(t)) .
\end{aligned}
$$

From the above discussion, the feedback controller $\boldsymbol{U}(t)$ can be designed as

$$
\boldsymbol{U}(t)=-\boldsymbol{\theta}(t)^{T} \boldsymbol{\varphi}(\boldsymbol{z}(t))-\boldsymbol{K} \operatorname{sign}(\boldsymbol{e}(t))-\boldsymbol{L}_{0} D_{t}^{\mu-1} \boldsymbol{e}(t)
$$

where $K=\operatorname{diag}\left[\hat{\varepsilon}_{1}^{*}(t), \hat{\varepsilon}_{2}^{*}(t), \ldots, \hat{\varepsilon}_{n}^{*}(t)\right], \hat{\varepsilon}_{i}^{*}(t)$ is the estimator of unknown constant $\varepsilon_{i}^{*}$, and $\boldsymbol{L}=\operatorname{diag}\left[l_{1}(t), l_{2}(t), \ldots, l_{n}(t)\right], l_{i}(t)(i=1,2, \ldots, n)$ is the estimator of feedback gain $l_{i}^{*}(>0)$. Then

$$
u_{i}(t)=-\theta_{i}(t)^{T} \varphi_{i}(\boldsymbol{z}(t))-\hat{\varepsilon}_{i}^{*}(t) \operatorname{sign}\left(e_{i}(t)\right)-l_{i}(t)\left({ }_{0} D_{t}^{\mu-1} e_{i}(t)\right)
$$

For achieving the synchronized target, we design an integer-order parameter adaptation, as follows:

$$
\begin{aligned}
& \dot{\theta}_{i}(t)=\lambda_{i}\left({ }_{0} D_{t}^{\mu-1} e_{i}(t)\right) \varphi_{i}(z(t))-\lambda_{i} \hat{\lambda}_{i} \theta_{i}(t) \\
& \dot{\hat{\varepsilon}}_{i}^{*}(t)=\xi_{i}\left({ }_{0} D_{t}^{\mu-1}\left|e_{i}(t)\right|\right)-\xi_{i} \hat{\xi}_{i} \hat{\varepsilon}_{i}^{*}(t) \\
& \dot{l}_{i}(t)=v_{i}\left({ }_{0} D_{t}^{\mu-1} e_{i}(t)\right)^{2}-v_{i} \hat{v}_{i} l_{i}(t)
\end{aligned}
$$

Here $\lambda_{i}, \hat{\lambda}_{i}, \xi_{i}, \hat{\xi}_{i}, v_{i}$ and $\hat{v}_{i}>0(i=1,2, \ldots, n)$ are designed parameters.

Remark 1 Comparing with the integer-order parameter adaptation in Ref. [49] that has the same aim as this study, the integer-order adaptive laws in the paper have been improved, adding a term (for example, the adaptive law (24) adds a term $-\xi_{i} \hat{\xi}_{i} \hat{\varepsilon}_{i}^{*}(t)$ ). We can easily learn from the integer-order adaptive laws in this paper: $\ddot{\hat{\varepsilon}}_{i}^{*}(t)=-\xi_{i} \hat{\xi}_{i}<0$, and $\hat{\varepsilon}_{i}^{*}(t)$ has a maximal value on $t \in[0,+\infty)$. Then $\hat{\varepsilon}_{i}^{*}(t)$ is bounded. Similarly, $\theta_{i}(t)$ and $l_{i}(t)$ are both bounded.

\section{Results and discussion}

We display some results in advance to facilitate the stability analysis of our control approach.

Lemma 3 If $e(t) \in R$, then ${ }_{0} D_{t}^{\mu-1}|e(t)| \geq 0$ and ${ }_{0} D_{t}^{\mu-1}|e(t)| \geq{ }_{0} D_{t}^{\mu-1} e(t)$. 
Proof According to the Caputo integro-differential definition, we have

$$
{ }_{0} D_{t}^{\mu-1}|e(t)|=\frac{1}{\Gamma(1-\mu)} \int_{0}^{t}(t-\tau)^{-\mu}|e(\tau)| d \tau .
$$

Because of $\Gamma(1-\mu) \geq 0, \tau \in\left[0, t^{-}\right]$and $(t-\tau)^{-\mu}|e(\tau)| \geq 0,{ }_{0} D_{t}^{\mu-1}|e(t)| \geq 0$.

Similarly, for

$$
{ }_{0} D_{t}^{\mu-1}(|e(t)|-e(t))=\frac{1}{\Gamma(1-\mu)} \int_{0}^{t}(t-\tau)^{-\mu}(|e(\tau)|-e(\tau)) d \tau
$$

obviously, $\Gamma(1-\mu) \geq 0$ and $(t-\tau)^{-\mu}(|e(\tau)|-e(\tau)) \geq 0$, then ${ }_{0} D_{t}^{\mu-1}(|e(t)|-e(t)) \geq 0$. From the linear property [44], we have ${ }_{0} D_{t}^{\mu-1}(|e(t)|-e(t))={ }_{0} D_{t}^{\mu-1}|e(t)|-{ }_{0} D_{t}^{\mu-1} e(t) \geq 0$. So ${ }_{0} D_{t}^{\mu-1}|e(t)| \geq{ }_{0} D_{t}^{\mu-1} e(t)$.

Lemma 4 If $e(t) \in C^{1}[0, h](h>0)$, then the following equality holds:

$$
\frac{d}{d t}\left({ }_{0} D_{t}^{\mu-1} e(t)\right)={ }_{0} D_{t}^{\mu} e(t)
$$

Proof Let $x(t)={ }_{0} D_{t}^{\mu-1} e(t)$, from Lemma 1, we can get

$$
{ }_{0} D_{t}^{1-\mu} x(t)=e(t)
$$

From Lemma 2, we know

$$
\frac{d x(t)}{d t}=\dot{x}(t)={ }_{0} D_{t}^{\mu} D_{t}^{1-\mu} x(t)={ }_{0} D_{t}^{\mu} e(t) .
$$

This ends the proof of Lemma 4.

Lemma 5 Suppose that $y(t)={ }_{0} D_{t}^{\mu-1} e(t)$ is asymptotically stable, i.e. $\lim _{t \rightarrow \infty} y(t)=0$, then $\lim _{t \rightarrow \infty} e(t)=0$.

Proof From Lemma 1, we have $e(t)={ }_{0} D_{t}^{1-\mu} y(t)$. Its Laplace transform is

$$
E(s)=s^{1-\mu} Y(s)-s^{-\mu} y(0),
$$

where $Y(s)$ and $E(s)$ are, respectively, the Laplace transforms of $y(t)$ and $e(t)$. According to the final value theorem of the Laplace transform of a continuous system, we have

$$
\lim _{t \rightarrow \infty} e(t)=\lim _{s \rightarrow 0} s E(s)=\lim _{s \rightarrow 0} s^{1-\mu}(s Y(s))-\lim _{s \rightarrow 0} s^{1-\mu} y(0) .
$$

Likewise, by the final value theorem: $\lim _{t \rightarrow \infty} y(t)=\lim _{s \rightarrow 0} s Y(s)=0$. So when $s \rightarrow 0, s Y(s)$ is an infinitesimal quantity. Since $s^{1-\mu}$ is also an infinitesimal quantity when $s \rightarrow 0$, we have $\lim _{s \rightarrow 0} s^{1-\mu}(s Y(s))=0$ and $\lim _{s \rightarrow 0} s^{1-\mu} y(0)=0$. Therefore $\lim _{t \rightarrow \infty} e(t)=\lim _{s \rightarrow 0} s E(s)=0$.

Lemma 6 Suppose that $y(t)={ }_{0} D_{t}^{\mu-1} e(t)$ is stable, then $e(t)$ is stable. 
Proof $y(t)$ is stable, i.e. for any $\varepsilon(>0)$, there exists $T_{0}$ such that

$$
-\varepsilon \leq{ }_{0} D_{t}^{\mu-1} e(t) \leq \varepsilon,
$$

for any $t \geq T_{0}$. Now, we use the right inequality of Eq. (33), whose left inequality can be used in the same way. Suppose that $0 \leq{ }_{0} D_{t}^{\mu-1} e(t) \leq \varepsilon$, there exists a nonnegative function $m(t)$ such that

$$
{ }_{0} D_{t}^{\mu-1} e(t)+m(t)=\varepsilon
$$

Taking the $\mu$ th integral of Eq. (34), we get $\int_{0}^{t} e(\tau) d \tau=\frac{\varepsilon t^{\mu}}{\mu \Gamma(\mu)}-{ }_{0} D_{t}^{-\mu} m(t)$. Owing to $m(t)$ being nonnegative, we have

$$
\int_{0}^{t} e(\tau) d \tau \leq \frac{\varepsilon t^{\mu}}{\mu \Gamma(\mu)}
$$

Setting the 1th derivative into inequality (35), we obtain

$$
e(t) \leq \frac{\varepsilon t^{\mu-1}}{\mu \Gamma(\mu)}
$$

In the same way as above, by the left inequality of Eq. (33), we obtain $-\frac{\varepsilon t^{\mu-1}}{\mu \Gamma(\mu)} \leq e(t)$, then we have

$$
|e(t)| \leq \frac{\varepsilon t^{\mu-1}}{\mu \Gamma(\mu)} .
$$

So, we see that $e(t)$ is stable.

Now, the main results of this paper can be concluded as the following theorem.

Theorem 2 The synchronization error between the drive system (13) and the response system (14) can converge to a small enough region of the origin by the action of adaptive fuzzy controller (21) together with integer-order adaptive laws (23), (24) and (25), and all the signals of the closed-loop system are bounded.

Proof Substituting controller (21) into the dynamic equation of error (16), we have

$$
{ }_{0} D_{t}^{\mu} \boldsymbol{e}(t)=\boldsymbol{F}(\boldsymbol{z}(t))-\boldsymbol{\theta}(t)^{T} \boldsymbol{\varphi}(\boldsymbol{z}(t))-\boldsymbol{K} \operatorname{sign}(\boldsymbol{e}(t))-\boldsymbol{L}_{0} D_{t}^{\mu-1} \boldsymbol{e}(t)
$$

It is simplified to

$$
{ }_{0} D_{t}^{\mu} \boldsymbol{e}(t)=-\tilde{\boldsymbol{\theta}}(t)^{T} \boldsymbol{\varphi}(\boldsymbol{z}(t))+\boldsymbol{\varepsilon}(\boldsymbol{z}(t))-\boldsymbol{K} \operatorname{sign}(\boldsymbol{e}(t))-\boldsymbol{L}_{0} D_{t}^{\mu-1} \boldsymbol{e}(t),
$$

where

$$
{ }_{0} D_{t}^{\mu} e_{i}(t)=-\tilde{\theta}_{i}(t)^{T} \varphi_{i}(\boldsymbol{z}(t))+\varepsilon_{i}(\boldsymbol{z}(t))-\hat{\varepsilon}_{i}^{*}(t) \operatorname{sign}\left(e_{i}(t)\right)-l_{i}(t){ }_{0} D_{t}^{\mu-1} e_{i}(t)
$$


Let $\tilde{\varepsilon}_{i}^{*}(t)=\hat{\varepsilon}_{i}^{*}(t)-\varepsilon_{i}^{*}, i=1,2, \ldots, n$. Consider the Lyapunov function as follows:

$$
\begin{aligned}
V(t)= & \frac{1}{2} \sum_{i=1}^{n}\left({ }_{0} D_{t}^{\mu-1} e_{i}(t)\right)^{2}+\frac{1}{2} \sum_{i=1}^{n} \frac{1}{\lambda_{i}} \tilde{\theta}_{i}(t)^{T} \tilde{\theta}_{i}(t) \\
& +\frac{1}{2} \sum_{i=1}^{n} \frac{1}{\xi_{i}}\left(\tilde{\varepsilon}_{i}^{*}(t)\right)^{2}+\frac{1}{2} \sum_{i=1}^{n} \frac{1}{v_{i}}\left(l_{i}(t)-l_{i}^{*}\right)^{2} .
\end{aligned}
$$

On the basis of Lemma 3 and Lemma 4, the derivative of Eq. (41) is

$$
\begin{aligned}
& \dot{V}(t)= \sum_{i=1}^{n}\left({ }_{0} D_{t}^{\mu-1} e_{i}(t)\right)_{0} D_{t}^{\mu} e_{i}(t)+\sum_{i=1}^{n} \frac{1}{\lambda_{i}} \tilde{\theta}_{i}(t)^{T} \dot{\tilde{\theta}}_{i}(t)+\sum_{i=1}^{n} \frac{1}{\xi_{i}} \tilde{\varepsilon}_{i}^{*}(t) \dot{\tilde{\varepsilon}}_{i}^{*}(t) \\
&+\sum_{i=1}^{n} \frac{1}{v_{i}}\left(l_{i}(t)-l_{i}^{*}\right) \dot{l}_{i}(t) \\
&=-\sum_{i=1}^{n}\left({ }_{0} D_{t}^{\mu-1} e_{i}(t)\right) \tilde{\theta}_{i}(t)^{T} \varphi_{i}(\boldsymbol{z}(t))+\sum_{i=1}^{n}\left({ }_{0} D_{t}^{\mu-1} e_{i}(t)\right) \varepsilon_{i}(\boldsymbol{z}(t)) \\
&-\sum_{i=1}^{n}\left({ }_{0} D_{t}^{\mu-1} e_{i}(t)\right) \hat{\varepsilon}_{i}^{*}(t) \operatorname{sign}\left(e_{i}(t)\right)-\sum_{i=1}^{n} l_{i}(t)\left({ }_{0} D_{t}^{\alpha-1} e_{i}(t)\right)^{2}+\sum_{i=1}^{n} \frac{1}{\lambda_{i}} \tilde{\theta}_{i}(t)^{T} \dot{\tilde{\theta}}_{i}(t) \\
&+\sum_{i=1}^{n} \frac{1}{\xi_{i}} \tilde{\varepsilon}_{i}^{*}(t) \dot{\tilde{\varepsilon}}_{i}^{*}(t)+\sum_{i=1}^{n} \frac{1}{v_{i}}\left(l_{i}(t)-l_{i}^{*}\right) \dot{i}_{i}(t) \\
& \leq-\sum_{i=1}^{n}\left({ }_{0} D_{t}^{\mu-1} e_{i}(t)\right) \tilde{\theta}_{i}(t)^{T} \varphi_{i}(z(t))-\sum_{i=1}^{n}\left({ }_{0} D_{t}^{\mu-1}\left|e_{i}(t)\right|\right) \tilde{\varepsilon}_{i}^{*}(t)-\sum_{i=1}^{n} \frac{1}{\xi_{i}} \tilde{\varepsilon}_{i}^{*}(t) \dot{\tilde{\varepsilon}}_{i}^{*}(t) \\
&+\sum_{i=1}^{n} \frac{1}{\lambda_{i}} \tilde{\theta}_{i}(t)^{T} \dot{\tilde{\theta}}_{i}(t)+\sum_{i=1}^{n} l_{i}(t)\left({ }_{0} D_{t}^{\mu-1} e_{i}(t)\right)^{2}+\sum_{i=1}^{n} \frac{1}{v_{i}}\left(l_{i}(t)-l_{i}^{*}\right) \dot{l}_{i}(t) \\
&= \sum_{i=1}^{n} \frac{1}{\lambda_{i}} \tilde{\theta}_{i}(t)^{T}\left[\dot{\tilde{\theta}}_{i}(t)-\lambda_{i}\left({ }_{0} D_{t}^{\mu-1} e_{i}(t)\right) \varphi_{i}(\boldsymbol{z}(t))\right] \\
&+\sum_{i=1}^{n} \frac{1}{\xi_{i}} \tilde{\varepsilon}_{i}^{*}(t)\left[\dot{\tilde{\varepsilon}}_{i}^{*}(t)-\xi_{i}\left({ }_{0} D_{t}^{\mu-1}\left|e_{i}(t)\right|\right)\right] \\
&+\sum_{i=1}^{n} \frac{1}{v_{i}}\left(l_{i}(t)-l_{i}^{*}\right)\left[\dot{l}_{i}(t)-v_{i}\left({ }_{0} D_{t}^{\mu-1} e_{i}(t)\right)^{2}\right]-\sum_{i=1}^{n} l_{i}^{*}\left({ }_{0} D_{t}^{\mu-1} e_{i}(t)\right)^{2} . \\
&(42) \\
&
\end{aligned}
$$

Substituting Eqs. (23)-(25) into Eq. (42), we get

$$
\begin{aligned}
\dot{V}(t) \leq & -\sum_{i=1}^{n} \hat{\lambda}_{i} \tilde{\theta}_{i}(t)^{T} \theta_{i}(t)-\sum_{i=1}^{n} \hat{\xi}_{i} \tilde{\varepsilon}_{i}^{*}(t) \hat{\varepsilon}_{i}^{*}(t)-\sum_{i=1}^{n} \hat{v}_{i}\left(l_{i}(t)-l_{i}^{*}\right) l_{i}(t) \\
& -\sum_{i=1}^{n} l_{i}^{*}\left({ }_{0} D_{t}^{\mu-1} e_{i}(t)\right)^{2} \\
\leq & -\sum_{i=1}^{n} l_{\min }\left({ }_{0} D_{t}^{\mu-1} e_{i}(t)\right)^{2}-\sum_{i=1}^{n} \hat{\lambda}_{i} \tilde{\theta}_{i}(t)^{T} \theta_{i}(t)-\sum_{i=1}^{n} \hat{\xi}_{i} \tilde{\varepsilon}_{i}^{*}(t) \hat{\varepsilon}_{i}^{*}(t)
\end{aligned}
$$




$$
\begin{aligned}
& -\sum_{i=1}^{n} \hat{v}_{i}\left(l_{i}(t)-l_{i}^{*}\right) l_{i}(t) \\
= & -\sum_{i=1}^{n} l_{\min }\left({ }_{0} D_{t}^{\mu-1} e_{i}(t)\right)^{2}-\sum_{i=1}^{n} \hat{\lambda}_{i} \tilde{\theta}_{i}(t)^{T} \tilde{\theta}_{i}(t)-\sum_{i=1}^{n} \hat{\xi}_{i}\left(\tilde{\varepsilon}_{i}^{*}(t)\right)^{2} \\
& -\sum_{i=1}^{n} \hat{v}_{i}\left(l_{i}(t)-l_{i}^{*}\right)^{2}-\sum_{i=1}^{n} \hat{\lambda}_{i} \tilde{\theta}_{i}(t)^{T} \theta_{i}^{*}-\sum_{i=1}^{n} \hat{\xi}_{i} \tilde{\varepsilon}_{i}^{*}(t) \varepsilon_{i}^{*}-\sum_{i=1}^{n} \hat{v}_{i} l_{i}^{*}\left(l_{i}(t)-l_{i}^{*}\right) \\
\leq & -\sum_{i=1}^{n} l_{\min }\left({ }_{0} D_{t}^{\mu-1} e_{i}(t)\right)^{2}-\sum_{i=1}^{n} \hat{\lambda}_{i} \tilde{\theta}_{i}(t)^{T} \tilde{\theta}_{i}(t)-\sum_{i=1}^{n} \hat{\xi}_{i}\left(\tilde{\varepsilon}_{i}^{*}(t)\right)^{2} \\
& -\sum_{i=1}^{n} \hat{v}_{i}\left(l_{i}(t)-l_{i}^{*}\right)^{2}+\sum_{i=1}^{n} \frac{\hat{\lambda}_{i}}{2}\left(\theta_{i}^{*}\right)^{T} \theta_{i}^{*}+\sum_{i=1}^{n} \frac{\hat{\xi}_{i}}{2}\left(\varepsilon_{i}^{*}\right)^{2}+\sum_{i=1}^{n} \frac{\hat{v}_{i}}{2}\left(l_{i}^{*}\right)^{2} \\
\leq & -a_{1} V(t)+a_{2},
\end{aligned}
$$

where $a_{1}=\min \left\{2 l_{\min }, 2 \lambda_{i} \hat{\lambda}_{i}, 2 \xi_{i} \hat{\xi}_{i}, 2 v_{i} \hat{v}_{i}\right\}$ and $a_{2}=\sum_{i=1}^{n} \frac{\hat{\lambda}_{i}}{2}\left(\theta_{i}^{*}\right)^{T} \theta_{i}^{*}+\sum_{i=1}^{n} \frac{\hat{\xi}_{i}}{2}\left(\varepsilon_{i}^{*}\right)^{2}+$ $\sum_{i=1}^{n} \frac{\hat{v}_{i}}{2}\left(l_{i}^{*}\right)^{2}$ are two positive constants.

It is known from Eq. (43) that there exists a nonnegative function $g(t)$ such that

$$
\dot{V}(t)+g(t)=-a_{1} V(t)+a_{2} .
$$

Taking the Laplace transform of Eq. (44), we obtain

$$
W(s)=\frac{V(0)}{s+a_{1}}+\frac{s^{-1} a_{2}}{s+a_{1}}-\frac{G(s)}{s+a_{1}},
$$

where $W(s)$ and $G(s)$ are the Laplace transforms of $V(t)$ and $g(t)$. According to Eq. (6), the solution of Eq. (45) is

$$
V(t)=\frac{V(0)}{e^{a_{1} t}}+\frac{a_{2}}{a_{1}}\left(1-\frac{1}{e^{a_{1} t}}\right)-g(t) * e^{-a_{1} t} .
$$

It is clear that $g(t)$ and $e^{-a_{1} t}$ are nonnegative functions such that $g(t) * e^{-a_{1} t} \geq 0$. Thereby, we get

$$
V(t) \leq \frac{V(0)}{e^{a_{1} t}}+\frac{a_{2}}{a_{1}}\left(1-\frac{1}{e^{a_{1} t}}\right) .
$$

Thus, we know that there exists $T_{0}$ such that

$$
\frac{V(0)}{e^{a_{1} t}}+\frac{a_{2}}{a_{1}}\left(1-\frac{1}{e^{a_{1} t}}\right) \leq \frac{\varepsilon}{2}+\frac{a_{2}}{a_{1}},
$$

for any $t \geq T_{0}$. If it makes the controller design parameter obey $\frac{a_{2}}{a_{1}} \leq \frac{\varepsilon}{2}$, then we can get

$$
V(t) \leq \varepsilon
$$

From Eqs. (49) and (41), we have $\left.{ }_{0} D_{t}^{\mu-1} e_{i}(t)\right)^{2} \leq 2 \varepsilon$ for all $t \geq T_{0}$. Then ${ }_{0} D_{t}^{\mu-1} e_{i}(t)$ is stable. According to Lemma 6 , we see that $e_{i}(t)$ is stable. Therefore, we see that $\boldsymbol{e}(t)$ is also stable. 
In addition, we see that $\dot{V}(t) \leq 0$ and $V(t)$ is monotone decreasing, i.e. $0 \leq V(t) \leq V(0)$. $V(t)$ is bounded. By Eq. (41), we know $\frac{1}{2} \sum_{i=1}^{n} \tilde{\theta}_{i}(t)^{T} \tilde{\theta}_{i}(t) \leq V(t) \leq V(0)$, i.e. $\tilde{\theta}_{i}(t)$ is bounded. Simultaneously, $\tilde{\varepsilon}_{i}(t)$ is also bounded. By Eq. (40), we have

$$
\left\|D_{0} D_{t}^{\mu} e_{i}(t)\right\| \leq\left\|\tilde{\theta}_{i}(t)^{T}\right\| \cdot\left\|\varphi_{i}(\boldsymbol{z}(t))\right\|+\left|\tilde{\varepsilon}_{i}^{*}(t)\right|+\left|l_{i}(t)\right| \cdot\left|{ }_{0} D_{t}^{\mu-1} e_{i}(t)\right| .
$$

So ${ }_{0} D_{t}^{\mu} e_{i}(t)$ is bounded. Since system (13) is a chaotic system, $\boldsymbol{x}(t)$ is bounded. In addition, $\boldsymbol{e}(t)$ is also bounded and $\boldsymbol{y}(t)$ is bounded. From the structure of the controller (21), we realize that $U(t)$ is also bounded. Therefore, all the signs of the closed-loop are bounded.

Remark 2 In order to guarantee the synchronization error to converge to a small enough region of the origin, from the proof process of Theorem 2 , we should make $a_{2} / a_{1}$ as small as possible via designing appropriate parameters of the fuzzy system, such as, we can choose larger $\lambda_{i}, \xi_{i}$ and $v_{i}$ and smaller $\hat{\lambda}_{i}, \hat{\xi}_{i}$ and $\hat{v}_{i}$.

Remark 3 Noting that besides the form of controller (22) being different from controller's in Ref. [49], it adds a feedback gain variable $l_{i}(t)$ that is automatically adjustable with the change of ${ }_{0} D_{t}^{\mu-1} e_{i}(t)$. And it strengthens the connection between $\boldsymbol{U}(t)$ and $\boldsymbol{e}(t)$ and makes the flexibility of $U(t)$ be more obvious.

Remark 4 According to Eq. (41) and inequalities (49), we see that $\|\tilde{\boldsymbol{\theta}}(t)\|^{2} \leq 2 V(t) \leq 2 \varepsilon$. Then we see that $\tilde{\theta}_{i}(t)$ can be arbitrarily small eventually. In the same way, $\tilde{\varepsilon}_{i}^{*}(t)$ is also arbitrarily small eventually. Besides, considering that ${ }_{0} D_{t}^{\mu-1} e_{i}(t)$ is stable and inequality (50), we can draw the extra conclusion that ${ }_{0} D_{t}^{\mu} e_{i}(t)$ is also arbitrarily small eventually. Therefore, we see that $\tilde{\theta}_{i}(t), \tilde{\varepsilon}_{i}^{*}(t)$ and ${ }_{0} D_{t}^{\mu} e_{i}(t)$ are all arbitrarily small eventually.

\section{Numerical simulation}

In this part, the effectiveness of the controller, which addresses the synchronization between an uncertain fractional-order Liu chaotic system with time-delay and an unknown fractional-order Chen chaotic system with time-delay, is tested by way of applying an improved prediction-correction [50].

The fractional-order Liu chaotic system with time-delay [51] is as follows:

$$
{ }_{0} D_{t}^{\alpha} \boldsymbol{x}(t)=\boldsymbol{f}\left(\boldsymbol{x}(t), \boldsymbol{x}\left(t-\tau_{1}\right)\right)=\left(\begin{array}{c}
10\left(x_{2}(t)-x_{1}\left(t-\tau_{1}\right)\right) \\
40 x_{1}\left(t-\tau_{1}\right)-x_{1}(t) x_{3}(t) \\
-2.5 x_{3}\left(t-\tau_{1}\right)+4 x_{1}^{2}(t)
\end{array}\right) .
$$

By Ref. [51], when $\alpha=0.97, \tau_{1}=0.005$ and initial value is $\boldsymbol{x}(0)=[2.2,2.4,3.8]^{T}$, system (51) shows chaotic phenomena. It is shown in Fig. 1.

The fractional-order Chen chaotic system with time-delay [52] is expressed as:

$$
{ }_{0} D_{t}^{\alpha} \boldsymbol{y}(t)=\boldsymbol{g}\left(\boldsymbol{y}(t), \boldsymbol{y}\left(t-\tau_{2}\right)\right)=\left(\begin{array}{c}
35\left(y_{2}(t)-y_{1}\left(t-\tau_{2}\right)\right) \\
-8 y_{1}\left(t-\tau_{2}\right)-y_{1}(t) y_{3}(t)+27 y_{2}(t) \\
-3 y_{3}\left(t-\tau_{2}\right)+y_{1}(t) y_{2}(t)
\end{array}\right) \text {. }
$$



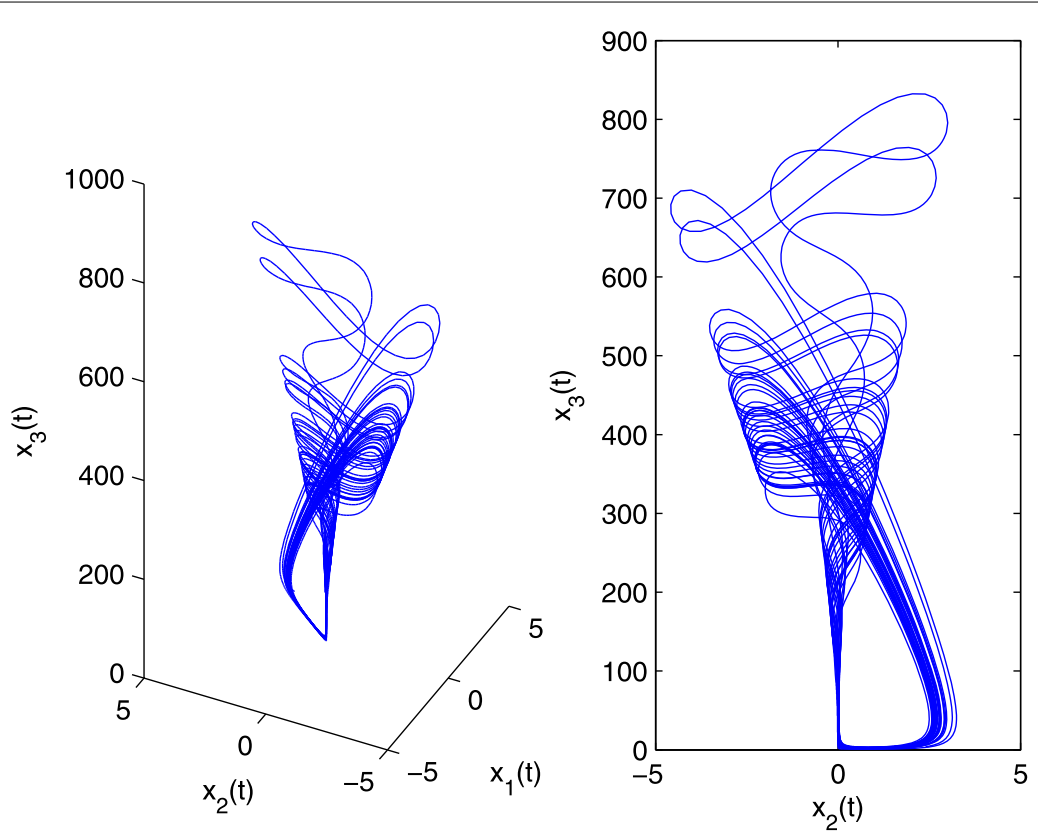

Figure 1 Fractional-order Liu system

Via Ref. [52], when $\alpha=0.97, \tau_{2}=0.009$ and initial condition is $\boldsymbol{y}(0)=[0.2,0,0.5]^{T}$, system (52) is in the chaotic state. It is displayed as Fig. 2.

The drive system, a fractional-order Liu system with time-delay, is

$$
\begin{aligned}
{ }_{0} D_{t}^{\alpha} \boldsymbol{x}(t)= & \boldsymbol{f}\left(\boldsymbol{x}(t), \boldsymbol{x}\left(t-\tau_{1}\right)\right)+\Delta \boldsymbol{h}_{1}(\boldsymbol{x}(t))+\boldsymbol{D}_{1}(t) \\
= & \left(\begin{array}{c}
10\left(x_{2}(t)-x_{1}\left(t-\tau_{1}\right)\right) \\
40 x_{1}\left(t-\tau_{1}\right)-x_{1}(t) x_{3}(t) \\
-2.5 x_{3}\left(t-\tau_{1}\right)+4 x_{1}^{2}(t)
\end{array}\right) \\
& +\left(\begin{array}{c}
0.03 \sin (\pi t) x_{2} \\
0.03 \sin (\pi t) x_{1} x_{3} \\
0.03 \sin (\pi t) x_{2}^{2}
\end{array}\right)+\left(\begin{array}{l}
0.1 \sin (t) \operatorname{rand}(t) \\
0.1 \sin (t) \operatorname{rand}(t) \\
0.1 \sin (t) \operatorname{rand}(t)
\end{array}\right) .
\end{aligned}
$$

The response system, a fractional-order Chen system with time-delay, is

$$
\begin{aligned}
{ }_{0} D_{t}^{\alpha} \boldsymbol{y}(t)= & \boldsymbol{g}\left(\boldsymbol{y}(t), \boldsymbol{y}\left(t-\tau_{2}\right)\right)+\Delta \boldsymbol{h}_{2}(\boldsymbol{y}(t))+D_{2}(t)+\boldsymbol{U}(t) \\
= & \left(\begin{array}{c}
35\left(y_{2}(t)-y_{1}\left(t-\tau_{2}\right)\right) \\
-8 y_{1}\left(t-\tau_{2}\right)-y_{1}(t) y_{3}(t)+27 y_{2}(t) \\
-3 y_{3}\left(t-\tau_{2}\right)+y_{1}(t) y_{2}(t)
\end{array}\right)+\left(\begin{array}{c}
0.02 \sin (2 \pi t) y_{2} \\
0.02 \sin (2 \pi t) y_{1} y_{3} \\
0.02 \sin (2 \pi t) y_{1} y_{2}
\end{array}\right) \\
& +\left(\begin{array}{c}
0.3 \sin (2 t) \operatorname{rand}(t) \\
0.3 \sin (2 t) \operatorname{rand}(t) \\
0.3 \sin (2 t) \operatorname{rand}(t)
\end{array}\right)+\left(\begin{array}{l}
u_{1}(t) \\
u_{2}(t) \\
u_{3}(t)
\end{array}\right) .
\end{aligned}
$$

In the simulation, the input variables of the fuzzy system are $\boldsymbol{x}(t), \boldsymbol{y}(t)$ and $\boldsymbol{U}(t)$. For reducing the calculation of the fuzzy logic system, we will let $\boldsymbol{x}(t), \boldsymbol{y}(t)$ be replaced by 

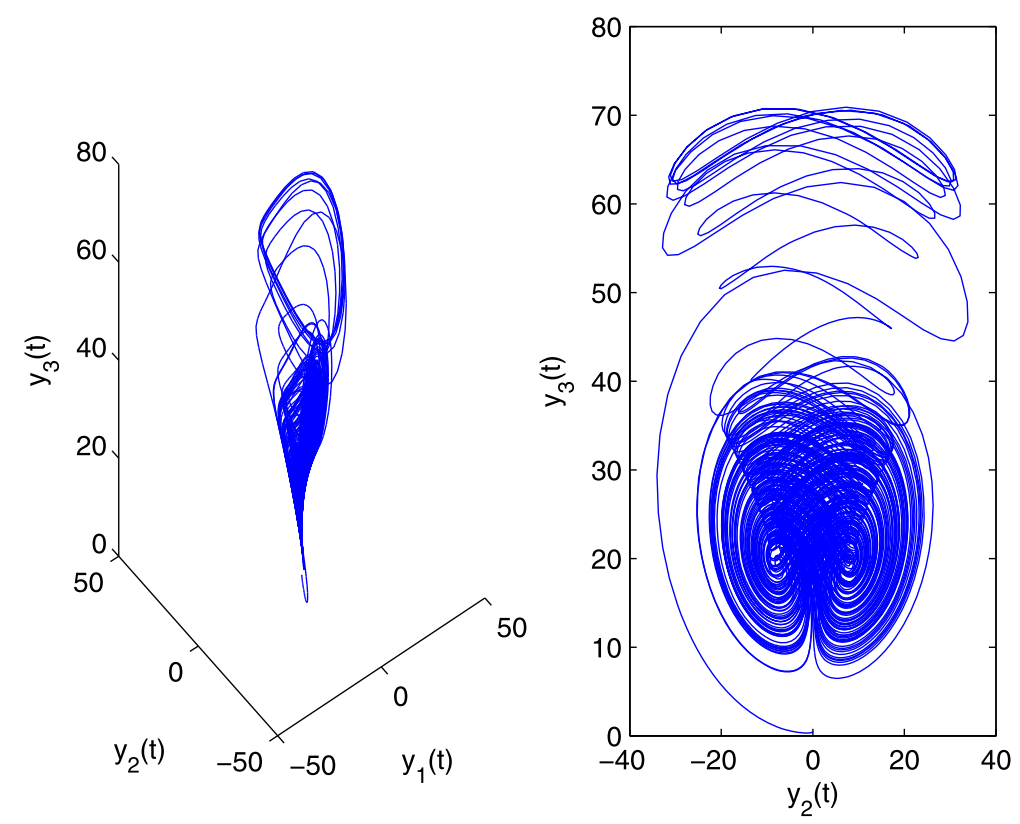

Figure 2 Fractional-order Chen system

$\boldsymbol{e}(t)$. For $e_{1}(t), e_{2}(t)$ and $e_{3}(t)$, we select five Gaussian membership functions, whose mathematical expectations are, respectively, $-5,-2.5,0,2.5$ and 5 and the parameters are $([1.2],[-5,-2.5,0,2.5,5])$, uniformly distributed on the interval $[-5,5]$ for each $e_{i}(t)$. Thereby, the number of the rules of fuzzy logic system approximating function is $5^{3}=125$. For the sake of better testing the effectiveness of the controller, we will define adjustable parameters, which are expressed by $\theta_{1}(0), \theta_{2}(0)$ and $\theta_{3}(0)$, as random vectors in 125 dimensions.

Other parameters of the controller are defined as $\lambda_{i}=30000, \xi_{i}=6, v_{i}=10, \hat{\lambda}_{i}=0.3, \hat{\xi}_{i}=$ 0.03 and $\hat{\nu}_{i}=0.05$, and the estimated values of fuzzy logic system approximating error are $\hat{\varepsilon}_{1}^{*}(0)=\hat{\varepsilon}_{2}^{*}(0)=\hat{\varepsilon}_{3}^{*}(0)=0.01$. The estimators of the feedback gain are $l_{1}(0)=l_{2}(0)=l_{3}(0)=5$ and the time interval is $h=0.005$. The simulation results are as in Fig. 4 and Fig. 3.

From the simulation results, we know that the synchronization errors in Fig. 3 are smaller and smaller after a short time and eventually converge to a small enough region. Moreover, we know the speed of error convergence is very fast, and it explains that the designed fuzzy logic system in this paper has good approximation performance. The changed situation displayed by Fig. 4, is that the tracking of state variables is basically consistent. The outcome of the simulation results conforms our expectation.

\section{Conclusions}

It can be seen that the designed adaptive feedback controller has strong anti-interference ability on the condition of not requiring an exact model and including time-delay state variables, unknown nonlinear terms and uncertain external disturbances. The stability of fractional-order chaotic systems with time-delays is successfully demonstrated via using integer-order derivatives of a quadratic Lyapunov function. We research fractional-order chaotic systems with different structures and time-delays with the help of the method of control and synchronization in an integer-order chaotic system. In the paper, the proposed 


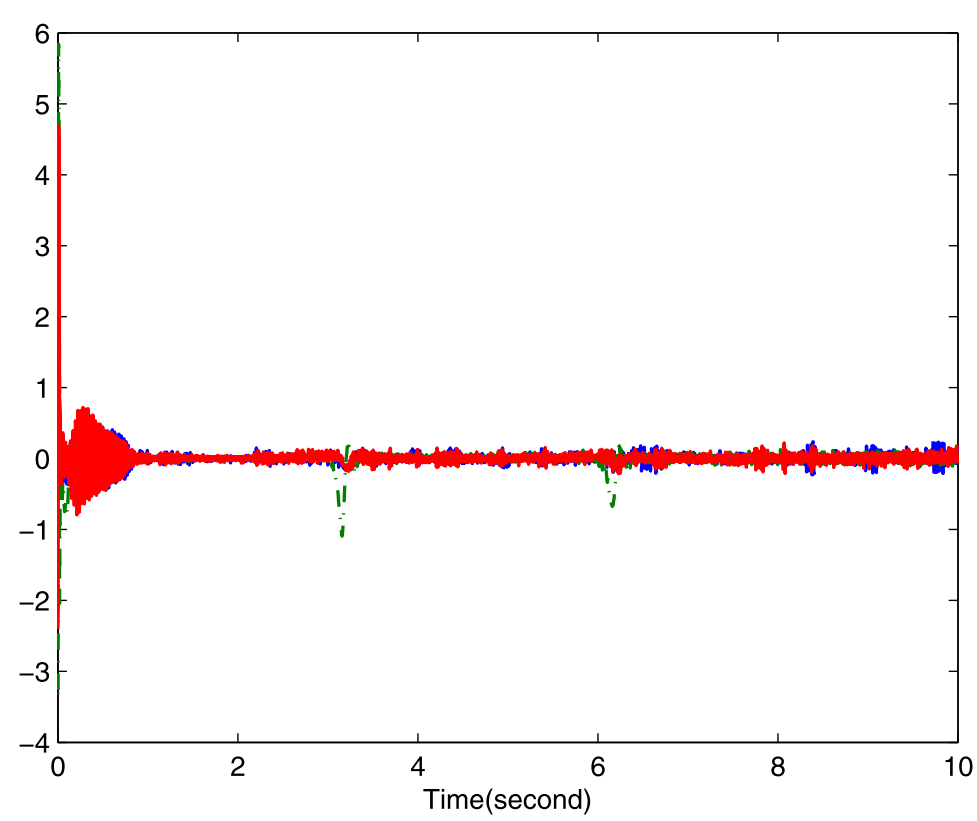

Figure 3 The synchronization error. $e_{1}(t)$ (Dotted line), $e_{2}(t)$ (chain line) and $e_{3}(t)$ (solid line)

(a)

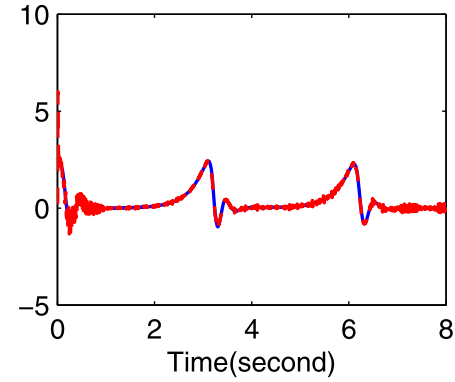

(c)

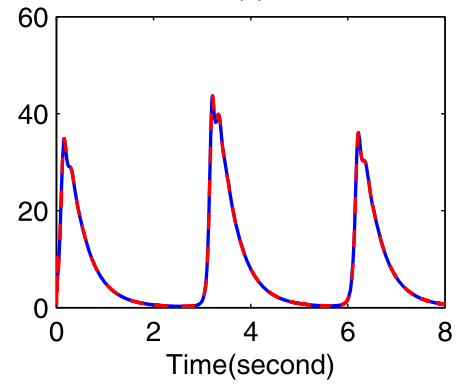

(b)

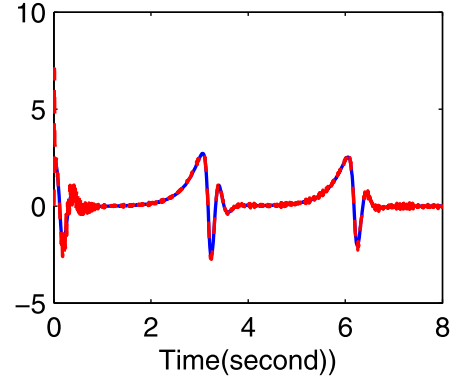

(d)

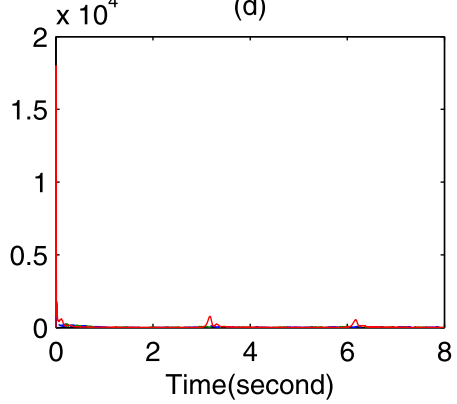

Figure 4 State variables and parameters of the fuzzy logic system. (a) $x_{1}(t)$ (Solid line) and $y_{1}(t)$ (dotted line), (b) $x_{2}(t)$ (solid line) and $y_{2}(t)$ (dotted line), (c) $x_{3}(t)$ (solid line) and $y_{3}(t)$ (dotted line) and (d) $\left\|\theta_{1}(t)\right\|$ (dotted line), $\left\|\theta_{2}(t)\right\|$ (chain line) and $\left\|\theta_{3}(t)\right\|$ (solid line)

method that considers the combination of integer-order and fractional-order derivatives solves the synchronization of fractional-order systems with time-delays. As a result, we can also consider that utilizing the proposed method solves the synchronization between integer-order and fractional-order systems with time-delays. Besides, it is still worth to 
researching whether we can consider the synchronization of uncertain fractional-order chaotic systems with different structures and time-delays by making use of fractionalorder parameter adaptive laws in the proposed method or by utilizing composite learning control [53].

\section{Acknowledgements}

Not applicable.

\section{Funding}

This work is supported by the National Natural Science Foundation of China (11771263), and the Natural Science Foundation of Anhui Province of China (1808085MF181).

\section{Competing interests}

The authors declare that they have no competing interests.

\section{Authors' contributions}

All authors contributed equally to the writing of this paper. All authors conceived of the study, participated in its design and coordination, read and approved the final manuscript.

\section{Author details}

'College of Mathematics and Information Science, Shaanxi Normal Universtiy, Xi'an, China. ${ }^{2}$ Deyang High School, Chengdu Normal Universtiy, Deyang, China. ${ }^{3}$ Department of Applied Mathematics, Huainan Normal University, Huainan, China.

\section{Publisher's Note}

Springer Nature remains neutral with regard to jurisdictional claims in published maps and institutional affiliations.

Received: 30 June 2018 Accepted: 24 April 2019 Published online: 08 May 2019

\section{References}

1. Li, Y., Chen, Y., Podlubny, I.: Mittag-Leffler stability of fractional order nonlinear dynamic systems. Automatica 45(8), 1965-1969 (2009)

2. Liu, H., Li, S., Wang, H., Sun, Y.: Adaptive fuzzy control for a class of unknown fractional-order neural networks subject to input nonlinearities and dead-zones. Inf. Sci. 454, 30-45 (2018)

3. Fernandez, A., Baleanu, D., Srivastava, H.: Series representations for fractional-calculus operators involving generalised Mittag-Leffler functions. Commun. Nonlinear Sci. Numer. Simul. 67, 517-527 (2019)

4. Wang, Y., Jiang, J.: Existence and nonexistence of positive solutions for the fractional coupled system involving generalized p-Laplacian. Adv. Differ. Equ. 2017(1), 337 (2017)

5. Feng, Q., Meng, F.: Traveling wave solutions for fractional partial differential equations arising in mathematical physics by an improved fractional Jacobi elliptic equation method. Math. Methods Appl. Sci. 40(10), 3676-3686 (2017)

6. Hao, X.: Positive solution for singular fractional differential equations involving derivatives. Adv. Differ. Equ. 2016(1), 139 (2016)

7. Zhu, B., Liu, L., Wu, Y.: Existence and uniqueness of global mild solutions for a class of nonlinear fractional reaction-diffusion equations with delay. Comput. Math. Appl. (2016, in press).

https://doi.org/10.1016/j.camwa.2016.01.028

8. Zhang, X., Liu, L., Wu, Y.: The uniqueness of positive solution for a fractional order model of turbulent flow in a porous medium. Appl. Math. Lett. 37, 26-33 (2014)

9. Zhang, L., Zheng, Z:: Lyapunov type inequalities for the Riemann-Liouville fractional differential equations of higher order. Adv. Differ. Equ. 2017(1), 270 (2017)

10. Li, M., Wang, J.: Exploring delayed Mittag-Leffler type matrix functions to study finite time stability of fractional delay differential equations. Appl. Math. Comput. 324, 254-265 (2018)

11. Liu, S., Wang, J., Zhou, Y., Fečkan, M.: Iterative learning control with pulse compensation for fractional differential systems. Math. Slovaca 68(3), 563-574 (2018)

12. Liu, H., Li, S., Li, G., Wang, H.: Adaptive controller design for a class of uncertain fractional-order nonlinear systems: an adaptive fuzzy approach. Int. J. Fuzzy Syst. 20(2), 366-379 (2018)

13. Muthukumar, P., Balasubramaniam, P., Ratnavelu, K.: Fast projective synchronization of fractional order chaotic and reverse chaotic systems with its application to an affine cipher using date of birth (dob). Nonlinear Dyn. 80(4), 1883-1897 (2015)

14. Tsirimokou, G., Psychalinos, C., Elwakil, A.: Design of CMOS Analog Integrated Fractional-Order Circuits: Applications in Medicine and Biology. Springer, Berlin (2017)

15. Yuan, L., Yang, Q.: Parameter identification of fractional-order chaotic systems without or with noise: reply to comments. Commun. Nonlinear Sci. Numer. Simul. 67, 506-516 (2019)

16. Liu, H., Pan, Y., Li, S., Chen, Y.: Adaptive fuzzy backstepping control of fractional-order nonlinear systems. IEEE Trans. Syst. Man Cybern. Syst. 47(8), 2209-2217 (2017)

17. Pan, Y., Er, M.J., Sun, T., Xu, B., Yu, H.: Adaptive fuzzy PD control with stable $h_{\infty}$ tracking guarantee. Neurocomputing 237, 71-78 (2017)

18. Dang, Q.A., Hoang, M.T.: Lyapunov direct method for investigating stability of nonstandard finite difference schemes for metapopulation models. J. Differ. Equ. Appl. 24(1), 15-47 (2018) 
19. Liu, H., Li, S.-G., Wang, H.-X., Li, G.-J: Adaptive fuzzy synchronization for a class of fractional-order neural networks. Chin. Phys. B 26(3), 030504 (2017)

20. Boulkroune, A., M'saad, M., Farza, M.: Adaptive fuzzy system-based variable-structure controller for multivariable nonaffine nonlinear uncertain systems subject to actuator nonlinearities. Neural Comput. Appl. 28(11), 3371-3384 (2017)

21. Liu, H., Li, S., Cao, J., Li, G., Alsaedi, A., Alsaadi, F.E.: Adaptive fuzzy prescribed performance controller design for a class of uncertain fractional-order nonlinear systems with external disturbances. Neurocomputing 219, 422-430 (2017)

22. Gao, L., Wang, D., Wang, G.: Further results on exponential stability for impulsive switched nonlinear time-delay systems with delayed impulse effects. Appl. Math. Comput. 268, 186-200 (2015)

23. Li, L., Meng, F., Ju, P.: Some new integral inequalities and their applications in studying the stability of nonlinear integro-differential equations with time delay. J. Math. Anal. Appl. 377(2), 853-862 (2011)

24. Cao, X., Wang, J.: Finite-time stability of a class of oscillating systems with two delays. Math. Methods Appl. Sci. 41(13), 4943-4954 (2018)

25. Yaghoobi, S., Parsa Moghaddam, B., Ivaz, K.: A numerical approach for variable-order fractional unified chaotic systems with time-delay. Comput. Methods Differ. Equ. 6(4), 396-410 (2018)

26. Huang, Y., Wang, D., Zhang, J., Guo, F.: Controlling and synchronizing a fractional-order chaotic system using stability theory of a time-varying fractional-order system. PLoS ONE 13(3), e0194112 (2018)

27. Li, H., Wang, J., Wu, L., Lam, H.-K., Gao, Y.: Optimal guaranteed cost sliding-mode control of interval type-2 fuzzy time-delay systems. IEEE Trans. Fuzzy Syst. 26(1), 246-257 (2018)

28. Pan, Y., Liu, Y., Xu, B., Yu, H.: Hybrid feedback feedforward: an efficient design of adaptive neural network control. Neural Netw. 76, 122-134 (2016)

29. Liu, H., Yang, J.: Sliding-mode synchronization control for uncertain fractional-order chaotic systems with time delay. Entropy 17(6), 4202-4214 (2015)

30. Li, D., Zhang, X.: Impulsive synchronization of fractional order chaotic systems with time-delay. Neurocomputing 216 39-44 (2016)

31. Wu, H.: Liouville-type theorem for a nonlinear degenerate parabolic system of inequalities. Math. Notes 103(1-2), 155-163 (2018)

32. Xu, R., Ma, X.: Some new retarded nonlinear Volterra-Fredholm type integral inequalities with maxima in two variables and their applications. J. Inequal. Appl. 2017(1), 187 (2017)

33. Peng, $X$., Shang, Y., Zheng, $X$.: Lower bounds for the blow-up time to a nonlinear viscoelastic wave equation with strong damping. Appl. Math. Lett. 76, 66-73 (2018)

34. Feng, D., Sun, M., Wang, X.: A family of conjugate gradient methods for large-scale nonlinear equations. J. Inequal. Appl. 2017(1), $236(2017)$

35. Li, F., Gao, Q.: Blow-up of solution for a nonlinear Petrovsky type equation with memory. Appl. Math. Comput. 274 383-392 (2016)

36. Lin, X., Zhao, Z.: Existence and uniqueness of symmetric positive solutions of $2 n$-order nonlinear singular boundary value problems. Appl. Math. Lett. 26(7), 692-698 (2013)

37. Li, Y., Tong, S.: Adaptive fuzzy control with prescribed performance for block-triangular-structured nonlinear systems. IEEE Trans. Fuzzy Syst. 26(3), 1153-1163 (2018)

38. Delavari, H., Ghaderi, R., Ranjbar, A., Momani, S.: Fuzzy fractional order sliding mode controller for nonlinear systems. Commun. Nonlinear Sci. Numer. Simul. 15(4), 963-978 (2010)

39. Mirzajani, S., Aghababa, M.P., Heydari, A.: Adaptive control of nonlinear fractional-order systems using t-s fuzzy method. Int. J. Mach. Learn. Cybern. 10(3), 527-540 (2019)

40. Kumar, V., Rana, K.: Nonlinear adaptive fractional order fuzzy pid control of a 2-link planar rigid manipulator with payload. J. Franklin Inst. 354(2), 993-1022 (2017)

41. Liu, Y.-J., Gong, M., Tong, S., Chen, C.P., Li, D.-J.: Adaptive fuzzy output feedback control for a class of nonlinear systems with full state constraints. IEEE Trans. Fuzzy Syst. 26(5), 2607-2617 (2018)

42. Li, Y., Sui, S., Tong, S.: Adaptive fuzzy control design for stochastic nonlinear switched systems with arbitrary switchings and unmodeled dynamics. IEEE Trans. Cybern. 47(2), 403-414 (2017)

43. Boulkroune, A.: A fuzzy adaptive control approach for nonlinear systems with unknown control gain sign. Neurocomputing 179, 318-325 (2016)

44. Pudlubny, I.: Fractional Differential Equations. Academic Press, San Diego (1999)

45. Haberman, R.: Applied Partial Differential Equations with Fourier Series and Boundary Value Problems. Pearson Higher Ed (2012)

46. Li, C., Deng, W.: Remarks on fractional derivatives. Appl. Math. Comput. 187(2), 777-784 (2007)

47. Boulkroune, A., Tadjine, M., M'Saad, M., Farza, M.: Fuzzy adaptive controller for mimo nonlinear systems with known and unknown control direction, Fuzzy Sets Syst. 161(6), 797-820 (2009)

48. Shaocheng, T., Jiantao, T., Tao, W.: Fuzzy adaptive control of multivariable nonlinear systems, Fuzzy Sets Syst., 111(2), 153-167 (2000)

49. Fei-Fei, L., Zhe-Zhao, Z:: Synchronization of uncertain fractional-order chaotic systems with time delay based on adaptive neural network control. Acta Phys. Sin. 66, 9 (2017)

50. Bhalekar, S., Daftardar-Gejji, V:: A predictor-corrector scheme for solving nonlinear delay differential equations of fractional order. J. Fract. Calc. Appl. 1(5), 1-9 (2011)

51. Bhalekar, S., Daftardar-Gejji, V:: Fractional ordered Liu system with time-delay. Commun. Nonlinear Sci. Numer. Simul. 15(8), 2178-2191 (2010)

52. Daftardar-Gejji, V., Bhalekar, S., Gade, P.: Dynamics of fractional-ordered Chen system with delay. Pramana 79(1), 61-69 (2012)

53. Pan, Y., Er, M.J., Liu, Y., Pan, L., Yu, H.: Composite learning fuzzy control of uncertain nonlinear systems. Int. J. Fuzzy Syst. 18(6), 990-998 (2016) 